\title{
BMJ Open Diagnostic accuracy of calculated serum osmolarity to predict dehydration in older people: adding value to pathology laboratory reports
}

Lee Hooper, ${ }^{1}$ Asmaa Abdelhamid, ${ }^{1}$ Adam Ali, ${ }^{1}$ Diane K Bunn, ${ }^{1}$ Amy Jennings, ${ }^{1}$ W Garry John, ${ }^{2}$ Susan Kerry, ${ }^{2}$ Gregor Lindner, ${ }^{3}$ Carmen A Pfortmueller, ${ }^{4}$ Fredrik Sjöstrand, ${ }^{5}$ Neil P Walsh, ${ }^{6}$ Susan J Fairweather-Tait, ${ }^{1}$ John F Potter, ${ }^{1,2}$ Paul R Hunter, ${ }^{1,2}$ Lee Shepstone ${ }^{1}$

To cite: Hooper L, Abdelhamid A, Ali A, et al. Diagnostic accuracy of calculated serum osmolarity to predict dehydration in older people: adding value to pathology laboratory reports. BMJ Open 2015;5:e008846. doi:10.1136/bmjopen-2015008846

- Prepublication history and additional material is available. To view please visit the journal (http://dx.doi.org/ 10.1136/bmjopen-2015008846).

Received 20 May 2015 Revised 26 August 2015 Accepted 18 September 2015

CrossMark

For numbered affiliations see end of article.

Correspondence to

Dr Lee Hooper;

L.hooper@uea.ac.uk

\section{ABSTRACT}

Objectives: To assess which osmolarity equation best predicts directly measured serum/plasma osmolality and whether its use could add value to routine blood test results through screening for dehydration in older people.

Design: Diagnostic accuracy study.

Participants: Older people ( $\geq 65$ years) in 5 cohorts: Dietary Strategies for Healthy Ageing in Europe (NU-AGE, living in the community), Dehydration Recognition In our Elders (DRIE, living in residential care), Fortes (admitted to acute medical care), Sjöstrand (emergency room) or Pfortmueller cohorts (hospitalised with liver cirrhosis).

Reference standard for hydration status: Directly measured serum/plasma osmolality: current dehydration (serum osmolality $>300 \mathrm{~m} 0 \mathrm{sm} / \mathrm{kg}$ ), impending/current dehydration ( $\geq 295 \mathrm{m0sm} / \mathrm{kg}$ ).

Index tests: 39 osmolarity equations calculated using serum indices from the same blood draw as directly measured osmolality.

Results: Across 5 cohorts 595 older people were included, of whom $19 \%$ were dehydrated (directly measured osmolality $>300 \mathrm{m0sm} / \mathrm{kg}$ ). Of 39 osmolarity equations, 5 showed reasonable agreement with directly measured osmolality and 3 had good predictive accuracy in subgroups with diabetes and poor renal function. Two equations were characterised by narrower limits of agreement, low levels of differential bias and good diagnostic accuracy in receiver operating characteristic plots (areas under the curve $>0.8$ ). The best equation was osmolarity $=1.86 \times\left(\mathrm{Na}^{+}+\mathrm{K}^{+}\right)+1.15 \times$ glucose + urea +14 (all measured in $\mathrm{mmol} / \mathrm{L}$ ). It appeared useful in people aged $\geq 65$ years with and without diabetes, poor renal function, dehydration, in men and women, with a range of ages, health, cognitive and functional status.

Conclusions: Some commonly used osmolarity equations work poorly, and should not be used. Given costs and prevalence of dehydration in older people we suggest use of the best formula by pathology laboratories using a cutpoint of $295 \mathrm{m0sm} / \mathrm{L}$ (sensitivity $85 \%$, specificity $59 \%$ ), to report dehydration risk opportunistically when serum glucose, urea and

\section{Strengths and limitations of this study}

- Dehydration has become a generic term - we have clearly described dehydration type (waterloss dehydration) and used serum osmolality, the correct reference standard.

- Assessment of equations in five different groups of older people including healthy free-living older people, frailer people living in residential care, and older people visiting emergency care or staying in hospital, living in several European countries, and including men and women, people with and without poor renal function, people with and without diabetes, with and without dehydration.

- Careful measurement of osmolality and the components of osmolarity in hospital laboratories with good standardisation or under research conditions, from community and hospital samples.

- Lack of incorporation of alcohol into the equations, though we did assess the effect of mild inebriation and found it only modestly affected results.

electrolytes are measured for other reasons in older adults.

Trial registration numbers: DRIE: Research Register for Social Care, 122273; NU-AGE: ClinicalTrials.gov NCT01754012.

\section{BACKGROUND}

Twenty per cent of older people living in UK residential care are dehydrated, ${ }^{1} 40 \%$ dehydrated on UK hospital admission ${ }^{2}$ and $>20 \%$ of free-living US older people ${ }^{3}{ }^{4}$ are dehydrated. Despite increased risks of disability, mortality and hospital admissions associated with water-loss dehydration (or simply dehydration, not to be confused with 
hypovolaemia, see box 1 for definitions $)^{5-7}$ dehydration is often unnoticed. Our aim was to add value to routine blood test results by using them to screen for dehydration in older people.

Water-loss dehydration is due to insufficient fluid intake and is characterised by an increase in directly measured (by freezing point depression) serum/plasma osmolality. Directly measured osmolality is the reference standard for hydration status in older people because osmolality is central to physiological fluid control, acting as a trigger for thirst and renal fluid conservation, ${ }^{10} \mathrm{a}$ single measurement can diagnose dehydration (without prior information), and other measures do not work well in older people. ${ }^{9} 11{ }^{12}$ Serum urea/creatinine ratio is non-specific and not useful in indicating hydration status when kidneys are not functioning well, common in older adults. ${ }^{9}{ }^{13}$ While low fluid intake is indicated in young adults by alterations in urinary parameters, ${ }^{14} 15$ actual dehydration appears to be better characterised even in young adults by plasma or serum osmolality, ${ }^{12}$ and in older adults urinary measures do not indicate hydration status as poor urinary concentration is common $^{16}{ }^{17}$ (L Hooper, DK Bunn, A Abdelhamid, et al. Dehydration assessed using urinary tests, how well do they work? Diagnostic accuracy in older people. Am J Clin Nutr 2015; submitted). Weight fluctuates in wellhydrated older people and dehydration may occur gradually rather than acutely, so sudden weight loss is not a good indicator. ${ }^{18} 19$ Physician assessment of hydration status is not consistent between physicians, ${ }^{20}$ and bioelectrical impedance does not appear accurate enough. ${ }^{21-23}$ For these reasons serum and plasma osmolality are stated as the reference standards for diagnosing water-loss dehydration in older adults. ${ }^{9} 122425$

\section{Box 1 Key concepts and definitions}

Dehydration: water-loss dehydration, the result of insufficient fluid intake, which leads to elevation of directly measured serum osmolality.

Directly measured serum osmolality. the osmotic concentration of blood serum, expressed as the number of milliosmoles of solute per kilogram of serum water. ${ }^{8}$ Directly measured serum osmolality is assessed by degree of freezing point depression. Normal values for directly measured serum osmolality, indicating euhydration, are 275 to $\leq 295 \mathrm{m0sm} / \mathrm{kg}$, while 295 to $300 \mathrm{m0sm} / \mathrm{kg}$ is classified as impending dehydration, and $>300 \mathrm{m0sm} / \mathrm{kg}$ as current dehydration. $^{9}$

Directly measured plasma osmolality. the osmotic concentration of blood plasma, expressed as the number of milliosmoles of solute per kilogram of plasma water. Directly measured plasma osmolality is assessed by degree of freezing point depression. Thought to be equivalent to directly measured serum osmolality. Calculated serum osmolarity. is an estimation of the osmolar concentration of serum and is proportional to the number of particles per litre of solution; it is expressed as $\mathrm{m0sm} / \mathrm{L}$. This is what is used when a calculated value is derived (for further details see Deardorff $^{8}$ ).
Monitoring directly measured serum osmolality will provide information on hydration status, but would be invasive, and is only partially automated in UK pathology laboratories so is expensive-growing numbers of requests for directly measured serum osmolality would cause sample handling problems in the clinical laboratory. Simple tests such as urine measures and skin turgor have not shown promise in screening for dehydration in older people ${ }^{1617}$ (L Hooper, et al. 2015, submitted), and calculated osmolarity is recommended in standard medical textbooks and doctors' websites to assess for dehydration. ${ }^{26-29}$ Components of osmolarity equations (sodium, potassium, urea and glucose) are commonly measured as part of routine blood tests when older people visit hospitals or general practitioners. Many osmolarity equations have been developed and are in use, but it is not clear which are most useful. In the Dehydration Recognition In our Elders (DRIE) study, which included older people living in UK residential care, we assessed diagnostic accuracy of different calculated osmolarity formulae, compared to directly measured serum osmolality and identified an osmolarity formula usefully diagnostic for dehydration. ${ }^{1}$ Calculated osmolarity $>296 \mathrm{mOsm} / \mathrm{L}$ had high sensitivity $(97 \%)$ and reasonable specificity $(76 \%)$, and a diagnostic OR of 99. A calculated osmolarity equation that accurately identifies dehydration in the full spectrum of older people (healthy older people, frailer people in residential care and those needing secondary care) could enable pathology laboratories to use routine blood tests to provide individual information on hydration status. This would enable health professionals to support older people to maintain or increase their fluid intake. We assessed which osmolarity equation best predicts directly measured serum/plasma osmolality (and dehydration) in five cohorts of older people, and whether it could be used to add value to routine blood test results through screening for dehydration in older people.

\section{METHODS \\ Datasets}

We assessed osmolarity equations in five data sets, each of which assessed directly measured osmolality, sodium, potassium, glucose and urea from a single blood draw for each participant. For each data set we removed participants aged $<65$ years, those missing any of serum/plasma osmolality, serum sodium, potassium, urea or glucose measurements or who presented values resulting from artefact or physiological extremes (potassium $>8 \mathrm{mmol} / \mathrm{L}$, sodium $<80 \mathrm{mmol} / \mathrm{L}, \quad$ osmolality $>340 \mathrm{mOsm} / \mathrm{kg}$ ). Estimated-glomerular filtration rate (eGFR) was calculated with the Modification of Diet in Renal Disease formula, ${ }^{30}$ truncated at 90 to reflect clinical practice. The reference standard was directly measured serum/plasma osmolality categorised as hydrated ( 275 to $<295 \mathrm{mOsm} / \mathrm{kg}$ ), impending dehydration $(295-300 \mathrm{mOsm} / \mathrm{kg})$ or current dehydration $(>300 \mathrm{mOsm} / \mathrm{kg}) .{ }^{9} 19$ 
The Dietary Strategies for Healthy Ageing in Europe (NU-AGE) study was a randomised controlled multicentre trial of healthy, independent older people (without frailty, heart failure or serious chronic illness) responsible for their own shopping/cooking/meal choice and preparation aged 65-79 years (http://www.nu-age.eu). The NU-AGE project aimed to assess effects of a 1 year dietary intervention on markers of inflammation and health. ${ }^{11} 31{ }^{32}$ We used cross-sectional baseline data from 271 Norfolk (UK) participants recruited between September 2012 and January 2014, of whom 238 had measured serum osmolality. Two were excluded (one potassium $>8 \mathrm{mmol} / \mathrm{L}$, one missing serum glucose), so 236 were included in analyses. Participants were asked to avoid alcohol for $24 \mathrm{~h}$ before phlebotomy, but this was not verified. Venepuncture was in the morning after $\geq 8 \mathrm{~h}$ fasting (though participants were encouraged to drink water). Whole blood was processed (using clot activator tubes) to give serum samples, and stored at $-80^{\circ} \mathrm{C}$ until analyses. Samples were analysed in the Department of Clinical Biochemistry, Norfolk and Norwich University Hospital (NNUH, Norfolk, UK). The laboratory is fully accredited (Clinical Pathology Accreditation), performs daily calibration, internal quality control and participates in External Quality Assessment. Serum osmolality was measured by depression of freezing point (Advanced model 2020 multisample osmometer; Advance Instruments, repeatability of $\pm 3 \mathrm{mOsm} / \mathrm{kg}$, SD 1 , in the $0-400 \mathrm{mOsm} / \mathrm{kg}$ region, coefficient of variance (CV) as for DRIE samples, $0.6 \%$ ), frozen samples defrosted at room temperature on a roller mixer. When sufficient blood was available we also assessed serum sodium and potassium (indirect ionselective electrode, ISE; Abbott Architect), urea (using urease; Abbott Architect), creatinine (enzymatic method; Abbott Architect), haemoglobin (Sysmex XN) and glucose (hexokinase/glucose 6-phosphate dehydrogenase; Abbott Architect). It is not known whether laboratory staff analysing directly measured serum osmolality were aware of other blood measurements or vice versa.

The DRIE cohort were aged $\geq 65$ years (range 65-105) living in residential care in Norfolk and Suffolk (UK), with a variety of cognitive and/or functional impairments. Those with heart failure, end-stage renal failure or terminal illness were excluded. ${ }^{1} 33$ Recruitment occurred between April 2012 and August 2013, and this analysis used baseline (cross-sectional) data. Full details of recruitment criteria, consent and the study flow have been published previously. ${ }^{1}$ Participants had low levels of self-reported alcohol intake, and none appeared inebriated on interview. During the interview non-fasting venous blood samples were collected from an antecubital vein or back of hand using needle and syringe after participants had rested sitting (occasionally lying) $\geq 5$ min. Samples were immediately transferred to SST vacutainers, stored at room temperature, delivered to the Department of Clinical Biochemistry (NNUH) within $4 \mathrm{~h}$ and analysed immediately for serum osmolality. Serum analyses for DRIE were as for NU-AGE (same laboratory, personnel, accuracy and equipment). We sent 19 hidden duplicate samples for serum osmolality analysis to the NNUH laboratory between June 2014 and January 2015 (samples taken from the same blood draw, but in separate tubes labelled with different sample numbers) to assess CV. The laboratory mean CV for these 19 duplicates was $0.6 \%$. Of 201 people living in residential care recruited and interviewed, 198 had directly measured serum osmolality, of whom 26 were missing serum glucose, so 172 were included in this analysis.

Fortes included people aged $\geq 60$ years admitted to Welsh acute medical care or emergency departments with any primary diagnosis and capacity to consent MayNovember 2011. ${ }^{17}$ Those too unwell, who had begun medical treatment or rehydration were excluded. Blood was collected from antecubital or dorsal metacarpal veins without venestasis into one lithium heparin coated vacutainer (Becton Dickinson, Oxford, UK) and centrifuged immediately $\left(1500 \mathrm{~g}, 10 \mathrm{~min}, 4^{\circ} \mathrm{C}\right)$. Plasma was aspirated and directly measured plasma osmolality assessed (freezing point depression osmometer, Model 330 MO; Advanced Instruments, Norwood, Massachusetts, USA) in duplicate. Where the difference was $<3 \mathrm{mOsm} / \mathrm{kg}$ the mean was used, otherwise further repeats were carried out until the mean was clear. The mean CV for the 2-8 duplications for each sample (mean 2.8 duplications) was $0.7 \%$. Standard solutions $(290 \mathrm{mOsm} / \mathrm{kg}$ ) were run daily to ensure $\pm 2 \mathrm{mOsm} / \mathrm{kg}$ precision. Serum sodium, potassium, urea and glucose were analysed in the hospital clinical biochemistry department (indirect ISE, Olympus AU 2700 automated chemistry immuno-analyser; Beckman Coulter, Brea, California, USA), so analysis of osmolarity components was blind to directly measured osmolality and vice versa. Participants were not asked about recent alcohol intake, but those clearly inebriated could not give informed consent. Of 180 participants recruited, one did not have plasma osmolality measured, 10 lacked sodium, 62 glucose and 10 were $<65$ years, leaving 97 analysed.

Sjöstrand recruited older adults ( $\geq 75$ years) able to provide informed consent and not critically ill who attended the emergency room of a Swedish tertiary care centre in spring-summer 2010. Those taking ACE inhibitors, $>40 \mathrm{mg} /$ day diuretics or $>50 \mathrm{mg} / \mathrm{d} \beta$-blockers were excluded, as were those with heart failure or under the influence of alcohol (assessed by study nurse, not discussed or tested). Main study results on fluid dynamics over several hours are not published, but some aspects have been reported. ${ }^{16}{ }^{34}$ This analysis used baseline directly measured serum osmolality and serum measures. Serum samples were analysed immediately at the Karolinska ISO-certified laboratory, osmolality measured using freezing point depression (Osmometer Advanced 2020, Advanced Instruments Inc, USA, CV unclear), serum sodium, potassium, urea, creatinine and glucose 
assessed (by indirect ISE, glucose assessment was duplicated and mean recorded, Hitachi 917, Naka, Japan). The osmometer was automated, so assessment of serum osmolality was independent of other blood measures. Of 41 older adults recruited, 5 were excluded (one had serum osmolality $>340 \mathrm{mOsm} / \mathrm{kg}$, two were missing potassium values and two urea), so 36 were included here.

Pfortmueller included adults admitted to a Swiss emergency department with primary diagnosis of decompensated liver cirrhosis, January 2002 to December 2012. ${ }^{35}$ Pfortmueller's retrospective analysis aimed to assess the association of glucose disturbances with outcome, and the select group included a high proportion with very low or raised non-fasting glucose. Patients were identified via computerised patient database (Qualicare Office, Medical Database Software; Qualidoc AG, Bern). Directly measured serum osmolality (Advanced 3900 osmometer, assessment of freezing point depression, $\mathrm{CV}<1 \%$ ) and electrolytes, urea and glucose (indirect ISE, Roche Modular 800 System) were measured by the Department of Clinical Chemistry, Bern University Hospital. Thirty-one participants were alcoholic, but recent alcohol intake was not recorded. It is unclear whether laboratory staff analysing directly measured serum osmolality were aware of other blood measures or vice versa. Of 312 participants in the data set, 58 were $\geq 65$ years, of whom we excluded four (one with osmolality $>340 \mathrm{mOsm} / \mathrm{kg}$, three potassium $>8 \mathrm{mmol} / \mathrm{L}$ ), therefore 54 were included in analyses.

\section{Osmolarity equations}

Fazekas et $a l^{36}$ collected 36 equations to calculate osmolarity. Since sodium, potassium, glucose and urea are regularly measured in older people undergoing blood tests, we focused on the 33 equations that only included these factors (omitting 3 equations including ionised calcium or lactate) $^{3738}$ (VA Nelson, R A Scheidt. Personal communication to Fazekas et al 2013, 1969). Fazekas multiplied results of several equations by 0.985 (as they were reported in $\mathrm{mOsm} / \mathrm{L}^{39-41}$ ); however, as this may not have been the original authors' intention we ran these equations with and without this multiplication (adding equations 25a and 27a). We also evaluated equations we have observed local physicians using including the Wikipedia equation, ${ }^{26}$ US National Health and Nutrition Examination Survey, ${ }^{42}$ MDcalc and Joint British Diabetes Societies equations ${ }^{27} 29$ and tonicity (associated with adverse outcomes ${ }^{6}$ ) (see online supplementary table S1). We assessed equivalence of each of the 39 calculated osmolarity equations to the reference standard.

\section{Terminology and units}

Directly measured osmolality was assessed in molal units $(\mathrm{mOsm} / \mathrm{kg})$ and calculated osmolarity produced molar units $(\mathrm{mOsm} / \mathrm{L})$ making terminology comparing the two measurements complex. For clarity we have written all equations using SI units, referred to as calculated osmolarity, and expressed in mOsm/L. Directly measured osmolality was measured and reported in $\mathrm{mOsm} / \mathrm{kg}$, while serum sodium, potassium, urea and glucose measurements were in $\mathrm{mmol} / \mathrm{L}$. Since we were aiming for equivalence between osmolarity and osmolality, units for the osmolar gap, the difference between directly measured osmolality and calculated osmolarity, were labelled mOsm. $^{43}$

\section{Statistical analysis}

We used descriptive statistics to summarise each cohort, and Pearson's correlation to assess associations between directly measured osmolality and serum sodium, potassium, urea, creatinine and glucose. Osmolarity was calculated using each of the 39 formulae for each participant and compared against directly measured osmolality (difference in mOsm, measured osmolality in $\mathrm{mOsm} / \mathrm{kg}$ minus calculated osmolarity in $\mathrm{mOsm} / \mathrm{L}$ ), then averaged for each equation in each cohort. We were interested in osmolarity equations which approximated directly measured osmolality, so we identified equations where:

1. Mean difference was -1 to $+1 \mathrm{mOsm}$;

2. There was no statistically significant difference between osmolarity equation results and directly measured osmolality ( $p$ value for the paired t test $\geq 0.01$, set at 0.01 due to multiple testing and aiming not to lose potentially useful equations at the beginning of the selection process).

We were interested in equations where $\geq 3$ of the 5 cohorts fulfilled either of these criteria.

Having chosen the five most useful equations this way, remaining analyses used only these equations. We assessed percentage of participants whose osmolarity equation results fell within $2 \%$ of directly measured osmolality for each cohort and used a weighted mean to assess equations across all cohorts and for specific subgroups. Bland-Altman plots compared each osmolarity equation with directly measured osmolality, plotting the difference against the mean of osmolality and osmolarity. To assess differential bias, Pearson's correlation assessed the association of the difference with osmolality, biochemical parameters (haemoglobin, sodium, potassium, glucose, urea and eGFR), age and measures of nutritional, cognitive and functional status. We created receiver operating characteristic (ROC) plots to compare the ability of each of the five equations to diagnose current dehydration (serum/ plasma osmolality $>300 \mathrm{mOsm} / \mathrm{kg}$ ). Sensitivity and specificity, positive and negative likelihood ratios were calculated for each equation compared to current dehydration, assessing specificity where sensitivity was $\geq 75 \%, \geq 80 \%, \geq 85 \%$ and $\geq 90 \%$, and assessing the sensitivity and specificity of an appropriate whole-number cutpoint. A decision threshold was determined using the method of Zweig and Campbell, ${ }^{44}$ calculating a slope $\mathrm{m}=$ (false-positive cost/ false-negative cost $) \times(1$-dehydration prevalence $) /($ dehydration prevalence). The best decision threshold was the point on the ROC curve where the line with this slope was tangent. All statistical analyses were carried out in excel or 
in STATA (IC 11.2), and statistical significance set at $\mathrm{p}<0.05$ unless otherwise stated. This paper conforms to STARD reporting standards for diagnostic studies. ${ }^{45}$

\section{RESULTS}

Participants' are characterised by cohort in table 1 . Briefly, NU-AGE participants (mean age 70 years) had good cognitive and functional status, and few participants had raised sodium, potassium, glucose or low eGFR. DRIE care home residents were older (mean 86 years), with lower cognitive and functional status, $>40 \%$ had poor renal function $($ eGFR $<60), 20 \%$ raised glucose. Pfortmueller participants were relatively young (mean 69 years, similar to NU-AGE), with high proportions of hyperglycaemia and hyperkalaemia, while Sjöstrand emergency department participants were older (mean 84 years, similar to DRIE) with high levels of dehydration and hypernatraemia. Fortes participants (mean 79 years) had the lowest levels of dehydration, but $36 \%$ had poor renal function. Current dehydration (directly measured serum/plasma osmolality $>300 \mathrm{mOsm} / \mathrm{kg}$ ) varied from $8 \%$ of participants (Fortes) to $44 \%$ (Sjöstrand).

Adverse events associated with blood draws include bruising, but no cohorts recorded bruising or noted other adverse effects of participation.

In all cohorts apart from Pfortmueller, sodium concentrations were strongly, statistically significantly correlated with serum/plasma osmolality (see online supplementary table S2). Pfortmueller data showed no statistically significant relationships between sodium, potassium, urea, creatinine or glucose and directly measured osmolality. Urea was less strongly but significantly correlated with directly measured osmolality. Potassium and creatinine were weakly and significantly or borderline significantly correlated with directly measured osmolality in NU-AGE, DRIE and Fortes, but not Sjöstrand or Pfortmueller. Glucose was weakly and significantly correlated with directly measured serum osmolality in DRIE, but not significantly in other cohorts. This suggested that useful osmolarity equations would probably include sodium, urea or creatinine and probably potassium and glucose. Participants with raised serum sodium, potassium, urea and glucose were classified by hydration status in online supplementary table S3.

\section{Absolute bias}

The difference (directly measured osmolality, $\mathrm{mOsm} / \mathrm{kg}$, minus calculated osmolarity, $\mathrm{mOsm} / \mathrm{L}$ ) varied from $-37.6 \mathrm{mOsm}$ (Fortes equation 27) to $31.8 \mathrm{mOsm}$ (NU-AGE equation 1). The $\mathrm{p}$ values (paired t test) comparing osmolarity and osmolality are displayed in online supplementary table S4. We were interested in equations where for $\geq 3$ of 5 cohorts the mean difference was -1 to +1 or the $p$ value was $\geq 0.01$. Equations which fulfilled the first criterion were 10, 24, 32 and 33, while equations 10, 24, 26, 32 and 33 fulfilled the second and were examined further.

\section{Predictive accuracy}

For these five equations we calculated percentage of participants whose osmolarity equation results fell within $2 \%$ of directly measured osmolality (see online supplementary figure S1). Percentages were $70-90 \%$ for most equations for NU-AGE, DRIE and Sjöstrand, but lower in Fortes (40-50\%) and Pfortmueller (30-50\%). We created a weighted mean percentage for each equation across all five cohorts, which confirmed equation 32 as consistently useful and returning a greater proportion of participants within $2 \%$ of directly measured osmolality.

Predictive accuracy was assessed for specific subgroups: people with and without diagnosed diabetes mellitus; with good or poor $($ eGFR <60) renal function; with normal hydration, impending or current dehydration; men and women; low-alcohol and high-alcohol intake (figure 1). Equations 10 (which did not include glucose) and 26 performed less well than other equations where participants were diabetic or had current dehydration. Equation 32 returned a higher proportion of participants within $2 \%$ of directly measured osmolality than the other equations for all subgroups, except of those who were without diabetes, well hydrated and currently dehydrated (when it was second most predictive).

\section{Bland-Altman analysis}

Bland-Altman plots compared the five osmolarity equations with directly measured osmolality, ${ }^{46}$ with $95 \%$ limits of agreement (see online supplementary figure S2). Narrower limits imply better agreement, and smaller mean difference suggests near equality of osmolality and osmolarity. The narrowest limits were provided by formula 32 for NU-AGE, DRIE and Fortes, and formula 32 was a close second to formula 33 for Sjöstrand, though not good for Pfortmueller. Equation 10 did not show the narrowest limits for any data set, equation 24 was joint equal for NU-AGE only, and equation 26 was narrowest for Pfortmueller.

\section{Differential bias}

For all five equations in all data sets the difference was positively associated with directly measured osmolality, although correlations were less strong for equations 32 and 33 (see online supplementary table S5). There was a tendency for the difference in equations 10, 24 and 26 to be significantly associated with sodium, potassium, urea, creatinine, glucose and eGFR values in several data sets (DRIE and Fortes in particular) while equations 32 and 33 appeared to be less related. No equations were consistently associated with age, body mass index, haemoglobin, cognitive or functional status.

\section{Diagnostic accuracy}

ROC plots compared ability of each equation to diagnose current dehydration (serum/plasma osmolality $>300 \mathrm{mOsm} / \mathrm{kg}$ ) (figure 2, see online supplementary table S6). Diagnostic accuracy is represented by area under the curve (AUC, greater AUC equating to greater 


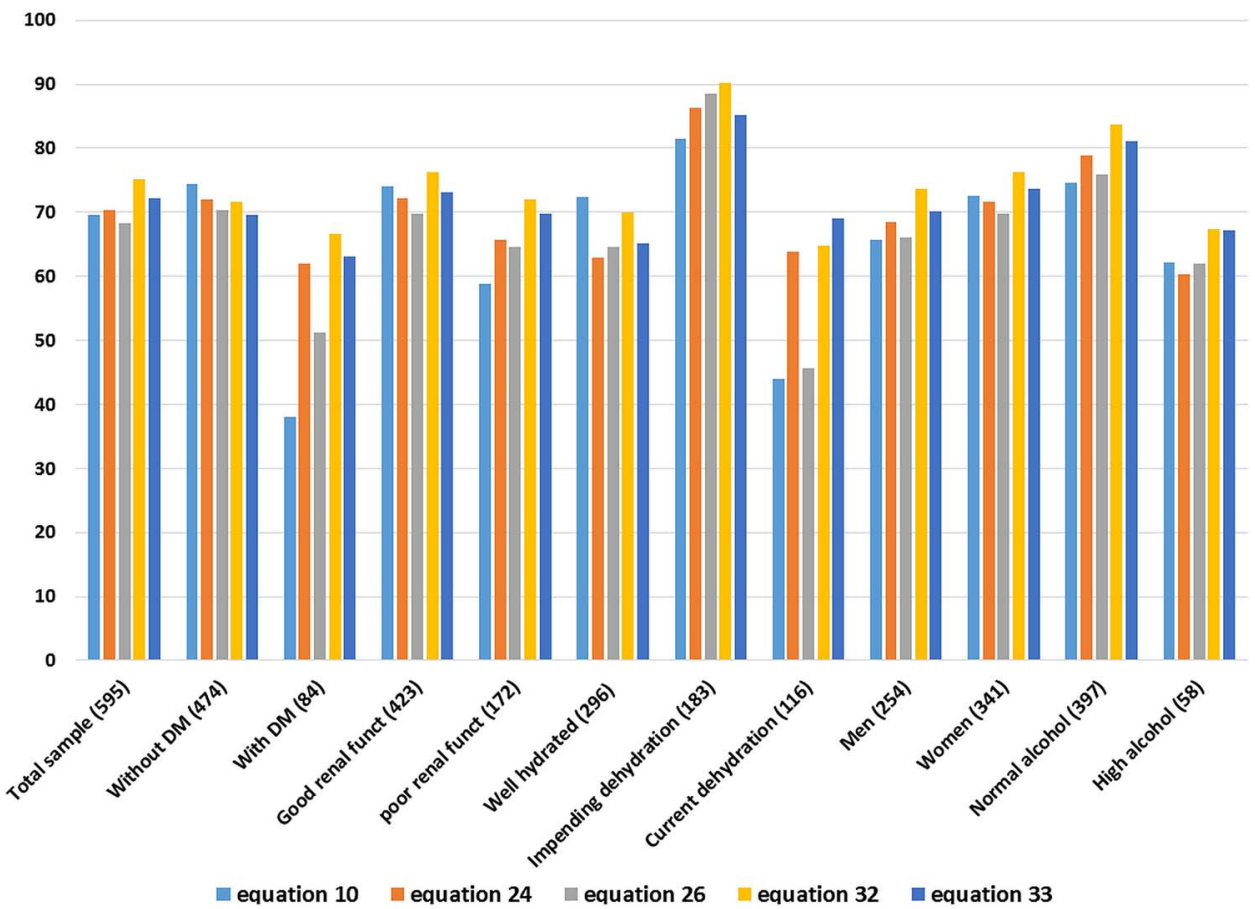

Figure 1 Percentages of individuals whose calculated osmolarity fell within $2 \%$ of measured osmolality, by equation and by specific conditions*. DM: diabetes mellitus. *In assessing by alcohol intake we had limited information on recent alcohol intake in any cohort, but Dehydration Recognition In our Elders (DRIE) participants reported very low alcohol intake, and the Dietary Strategies for Healthy Ageing in Europe (NU-AGE) participants had usual alcohol intake assessments so we separated out those who drank $\geq 21 \mathrm{~g}$ alcohol/week (intake mean plus one SD). For Pfortmueller we separated out alcoholics, other cohorts were not represented.

dehydration as assessed by equation $32 .{ }^{44}$ The mean prevalence of dehydration across all five cohorts was 0.19 , and we estimated that the cost of a false-negative finding (missing that a person is dehydrated, with its health consequences) has five-times the cost of a false-positive finding (labelling a person as dehydrated when they are not, resulting in a further blood test to directly measure osmolality or simply encouraging them to drink more).
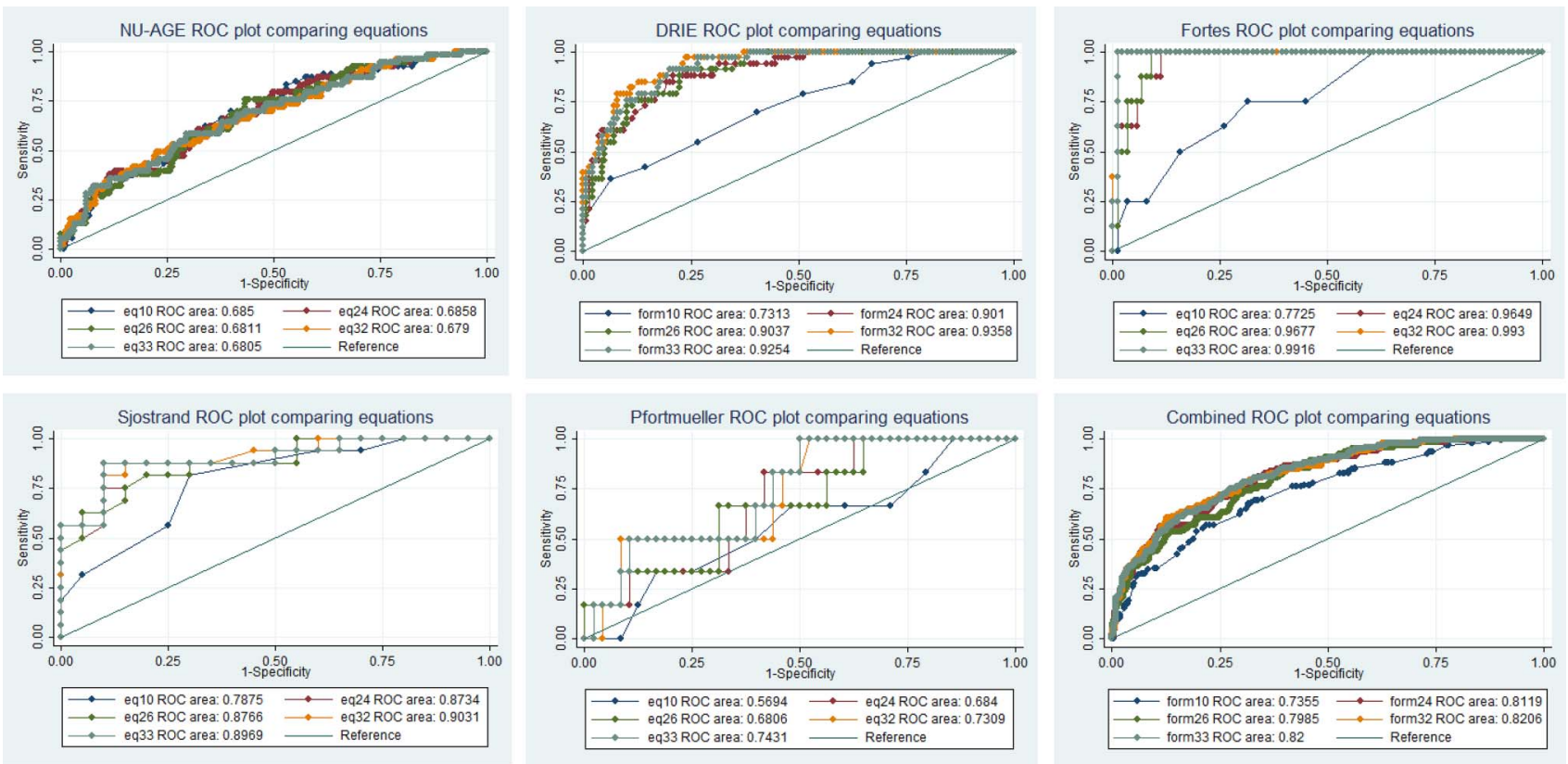

Figure 2 ROC plots for each equation for each data set and for all data sets combined. DRIE, Dehydration Recognition In our Elders; NU-AGE, the Dietary Strategies for Healthy Ageing in Europe; ROC, receiver operating characteristic. 
This gave a slope of $0.2 \times 0.81 / 0.19=0.85$. A line with a slope of 0.85 , is tangent to the equation 32 ROC curve at the cutpoint of $295 \mathrm{mOsm} / \mathrm{L}$ giving sensitivity of $84.5 \%$ and specificity of $58.9 \%$ (with a positive likelihood ratio of 2.05 and a negative likelihood ratio of 0.26 ).

\section{DISCUSSION}

We report the most comprehensive assessment of the relationship between calculated osmolarity and directly measured osmolality in older people to-date. One osmolarity equation (equation 32, by Khajuria and $\mathrm{Krahn}^{47}$ ) estimated directly measured serum/plasma osmolality well across healthy and frail older people, those in and out of hospital, with and without diabetes, with and without poor renal function, at all levels of directly measured serum osmolality (or dehydration) and in men and women. This equation (osmolarity $=1.86 \times\left(\mathrm{Na}^{+}+\mathrm{K}^{+}\right)$ $+1.15 \times$ glucose+urea+14) had $\mathrm{ROC}_{\mathrm{AUC}} 0.82(95 \%$ CI 0.78 to 0.86 ). Using an osmolarity cutpoint of $>295 \mathrm{mOsm} / \mathrm{L}$ gives sensitivity of $85 \%$ accompanied by specificity of $59 \%$ over the full range of older participants. Of the other, widely used, osmolarity equations (including equations suggested by standard medical sources) many were poor at predicting directly measured serum/ plasma osmolality, the reference standard for water-loss dehydration in older people. This suggests that some equations should not be used to estimate directly measured osmolality.

Study strengths include careful measurement of osmolality and the components of osmolarity in hospital laboratories with good standardisation or under research conditions (regular external standard, duplicating analyses of every sample).

A potential weakness was lack of incorporation of alcohol into the equations. Alcohol depresses serum/ plasma freezing point, increasing directly measured osmolality, with minimal impact on electrolytes, urea and glucose (increasing the difference between directly measured osmolality and osmolarity equations, the

Table 2 Summary of results of analyses by equation

\begin{tabular}{llllll}
\hline Test & Eq10 & Eq24 & Eq26 & Eq32 & Eq33 \\
\hline $\begin{array}{l}\text { Percentage of } \\
\text { participants with }\end{array}$ & $X$ & - & $X$ & & - \\
$\begin{array}{l}\text { osmolarity within 2\% } \\
\text { of osmolality }\end{array}$ & & & & & \\
$\begin{array}{l}\text { Bland-Altman } \\
\text { analyses }\end{array}$ & $X$ & - & - & & \\
$\begin{array}{l}\text { Differential bias } \\
\text { ROC plots }\end{array}$ & $X$ & $X$ & $X$ & $X$ & \\
$\begin{array}{l}\text { Sensitivity and } \\
\text { specificity }\end{array}$ & $X$ & - & $X$ & - & \\
\hline$X$, & & & & &
\end{tabular}

$\mathrm{X}$, indicates that a test is not useful; -, indicates a test which sometimes appears useful, but not consistently; that it does well and appears particularly useful.

ROC, receiver operating characteristic. osmolar gap). The full Khajuria and $\mathrm{Krahn}^{47}$ equation incorporates blood alcohol:

$$
\begin{aligned}
\text { Calculated osmolarity } & =1.86 \times(\mathrm{Na}+\mathrm{K})+1.15 \\
& \times \text { glucose }+ \text { urea }+1.2 \\
& \times \text { ethanol }+14
\end{aligned}
$$

(all components in $\mathrm{mmol} / \mathrm{L}$ )

No participants in any cohort were noticeably inebriated, so we estimated effects on calculated osmolarity of drinking 0.5 or 1 bottle of wine 2 or $8 \mathrm{~h}$ before phlebotomy (assuming $70 \mathrm{~kg}$ body weight). Blood alcohol was negligible to $2.8 \mathrm{mmol} / \mathrm{L}^{\mathrm{i}}$, highest in those drinking a bottle of wine $2 \mathrm{~h}$ before phlebotomy which increased calculated osmolarity by $3.4 \mathrm{mOsm} / \mathrm{L}$. This alters the relationship between osmolarity and directly measured osmolality by only a small amount. Our analyses suggested that equations' performance were slightly poorer in people more likely to have taken recent alcohol (figure 1), but this effect was not dramatic (and categories poorly defined). Despite likely alcohol intake in some participants osmolarity equations were good at screening for dehydration.

A further potential weakness was that we assumed that plasma osmolality (directly measured in Fortes) and serum osmolality (directly measured in the other cohorts) were equivalent. However, a high-quality study comparing lithium heparin plasma (as used in Fortes) with serum samples found equivalent directly measured osmolality. ${ }^{48}$ They also confirm that directly measured serum osmolality of fresh and frozen serum samples (stored at $-78^{\circ} \mathrm{C}$ and thawed rapidly) were equivalent (although not when stored at higher temperatures). ${ }^{48} 49$ On this basis we believe it is appropriate to use directly measured heparinised plasma osmolality and serum osmolality, as well as the osmolality of fresh and frozen samples (NU-AGE samples were the only frozen samples and were stored at $-80^{\circ} \mathrm{C}$ ), interchangeably. Previous studies have suggested a rise of $1-2 \mathrm{mOsm} / \mathrm{kg}$ in directly measured serum osmolality of samples stored at room temperature for up to $4 \mathrm{~h}$ before serum separation (as occurred in DRIE, and is standard practice for samples taken in primary care and transported to hospital laboratories for analysis). ${ }^{50}$

In all of our cohorts sodium and potassium concentrations were determined by indirect ISE (typically used in the large chemistry analysers in clinical laboratories, indirect ISE measures sodium on a plasma or serum sample that has been diluted with a large volume of diluent while direct ISE measures the electrolyte activity in the plasma water using a non-diluted whole-blood, plasma or serum sample and is typically used in point-of-care analysers)..$^{51}$ Indirect ISE (unlike direct ISE) is affected by the volume of non-aqueous cell components, lipids and proteins. While samples with high

${ }^{\mathrm{i}}$ http://www.csgnetwork.com/bloodalcolevelcalc.html 
levels of lipid or protein are routinely re-analysed using direct ISE, low protein concentrations, common in older adults, may lead to higher sodium and potassium readings (compared to direct ISE), ${ }^{52}$ affecting $25 \%$ of intensive care unit samples and $8 \%$ of general hospital samples. While this may be reflected in some imprecision in the matching of osmolarity formulae to directly measured osmolality our results suggest that despite use of indirect ISE the Khajuria and Krahn equation is useful in screening for dehydration in older people.

The Khajuria and Krahn equation is generalisable across healthy free-living older people, frailer people living in residential care, and older people visiting emergency care or staying in hospital. It worked well in those with and without good renal function, with and without diabetes, with and without dehydration, with and without a tendency to drink alcohol and in men and women. It even worked well in patients with decompensated liver cirrhosis (who experience difficulty with sodium and water balance due to abnormalities in antidiuretic hormone and aldosterone, reflected in low mean sodium). It works well using standard hospital equipment for analysis of sodium, potassium, urea and glucose, in plasma and serum samples, in fresh and frozen samples.

Classical thought is that hypernatraemia principally explains raised osmolality when fluids are restricted, ${ }^{53}$ and the correlations in online supplementary table S2 were highest between serum osmolality and sodium, but there were also significant correlations with potassium, urea, creatinine and glucose in some cohorts, suggesting that these also contribute. The statistical significance of correlations in different cohorts may partially relate to cohort characteristics-we are more likely to see weak but statistically significant relationships in the larger cohorts (NU-AGE and DRIE). In NU-AGE there was no correlation between glucose and serum osmolality, but only $1 \%$ of NU-AGE participants had raised serum glucose, while in DRIE, where $20 \%$ of participants had raised serum glucose, the relationship with osmolality was weak but statistically significant. Raised serum sodium was not equivalent to raised serum osmolality in these cohorts of older people (only $4 \%$ had serum sodium $>145 \mathrm{mmol} / \mathrm{L}$, while $19 \%$ had serum osmolality $>300 \mathrm{mOsm} / \mathrm{kg}$, see online supplementary table S3). This reflects data in young fit army volunteers dehydrated by walking and fluid restriction in a hot environment, where only one of 36 volunteers who were clearly dehydrated (fluid loss assessed by weight loss) had raised serum sodium-plasma osmolality, with a threshold of $301 \mathrm{mOsm} / \mathrm{kg}$, reflected hydration status much better than serum sodium. ${ }^{25}$ It appears that to assess hydration status plasma or serum osmolality is key, and to estimate serum osmolality well in cohorts with a variety of characteristics the contribution of sodium, potassium, glucose and urea are all crucial.

The finding that the Khajuria and $\mathrm{Krahn}^{47}$ equation (equation 32) was most useful in older people is consistent with our findings in DRIE data alone, ${ }^{1}$ but the equation may also be useful in younger populations. Heavens $e t a \bar{l}^{4}$ recently assessed a similar set of osmolarity equations against directly measured plasma osmolality in 60 young volunteers (aged 19-46 years), and found that five equations were useful, including Khajuria and Krahn's. ${ }^{47}$ The suggestion that this formula may be useful in young fit adults as well as in older adults adds weight to its utility.

Pathology laboratories could use this equation to report calculated osmolarity and hydration status of older people when analysing any blood sample including sodium, potassium, urea and glucose. For such routine screening for dehydration we could choose a cutpoint of $\geq 295 \mathrm{mOsm} / \mathrm{L}$ to provide sensitivity of $85 \%$ and specificity of $59 \%$, where a positive finding could be followed up by directly measured serum osmolality to confirm diagnosis and rule out false positives (suggested proforma in figure 3). A finding of dehydration would be followed by consideration of glucose-if glucose is raised then treatment would address serum glucose (across our populations 16\% of those with raised directly measured serum osmolality had raised glucose). Where glucose is not raised treatment would focus on increasing fluid intake. This screening would allow for early identification of dehydration (but not hypovolaemia) in older people, at little additional cost to the National Health Service. The alternative would be to assess for dehydration by directly measuring serum osmolality across the older population, but the cost of this, in laboratory time, resource and expense would be prohibitive without additional resources.

Using routine blood tests in older people to screen for dehydration using the Khajuria and Krahn formula for serum osmolarity would enable healthcare professionals and carers to provide appropriate support in older people by increasing fluid intake and improving and maintaining good hydration and thereby prevent associated poor health. This information could be provided automatically on the reports from pathology laboratories where serum sodium, potassium, urea and

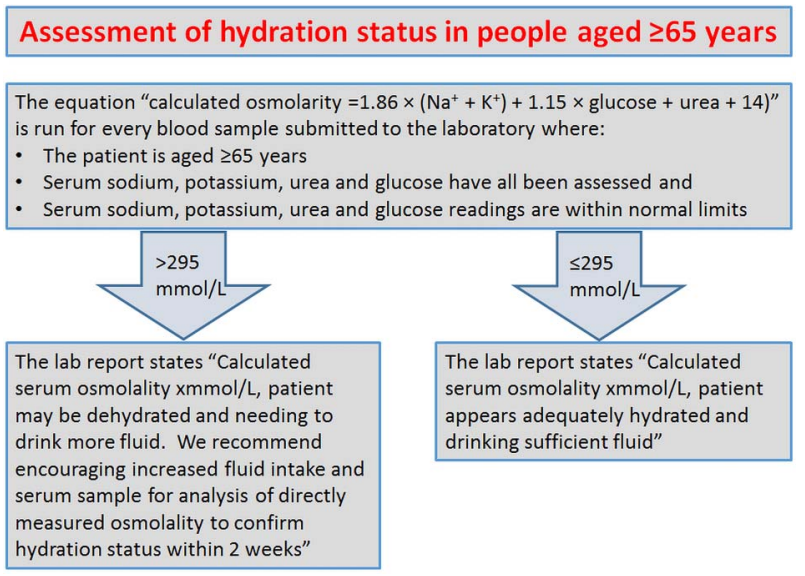

Figure 3 Suggested proforma for opportunistic assessment of hydration status by health laboratories. 
glucose have been measured, although to improve sensitivity (though increasing costs) positive results from this screening could be followed by assessment of directly measured serum osmolality. To assess the costeffectiveness of these different screening models we need detailed data on the costs of dehydration and of serum osmolality analysis.

\section{Author affiliations \\ ${ }^{1}$ Norwich Medical School, University of East Anglia, Norwich, UK \\ ${ }^{2}$ Norfolk and Norwich University Hospitals NHS Foundation Trust, Norwich, UK ${ }^{3}$ Department of General Internal Medicine, Inselspital, University Hospital Bern, Bern, Switzerland \\ ${ }^{4}$ Department of General Anesthesiology, Intensive Care and Pain Management, Medical University of Vienna, Vienna, Austria \\ ${ }^{5}$ Department of Emergency Medicine, Södersjukhuset AB, Stockholm, Sweden \\ ${ }^{6}$ College of Health and Behavioural Sciences, Bangor University, Bangor, UK}

Acknowledgements The authors would like to thank and acknowledge the participants of the DRIE and NU-AGE, Fortes, Sjöstrand and Pfortmueller studies, and DRIE advisory and steering group members, for their help, time, enthusiasm, support, ideas and participation with this work.

Contributors The DRIE study was conceived by $\mathrm{LH}$, and developed by $\mathrm{LH}$, DKB, JP, PRH and LS. NU-AGE was conceived by SJF-T and developed by AJ and SJF-T. The Fortes study was overseen by NPW, Sjöstrand by FS, Pfortmueller by CAP and GL. This analysis was conceived by LH, carried out by $\mathrm{LH}$ and $\mathrm{AA2}$ and developed by all authors. Data collection was carried out by LH, DKB, AJ, CAP, FS and LS. WGJ and SK oversaw analysis of samples for DRIE and NU-AGE and discussed laboratory implications. LH, AA1 and DKB wrote the first draft of the manuscript, all authors revised it critically for important intellectual content and agreed the final draft. All authors declare themselves to be accountable for all aspects of the work in ensuring that questions related to the accuracy or integrity of any part of the work are appropriately investigated and resolved.

Funding This paper summarises independent research funded in part by NHS England, in part by the National Institute of Health Research Fellowship programme (DRIE data, NIHR-CDF-2011-04-025), the European Union's Seventh Framework Programme under grant agreement no. 266486 ('NU-AGE: New dietary strategies addressing the specific needs of the elderly population for healthy ageing in Europe'), HydraDx Inc. (Fortes data), and a regional agreement on medical training and clinical research (ALF) between Stockholm County Council and Karolinska Institutet and an unrestricted grant by Masimo Inc, Irvine, CA (Sjöstrand data). The views expressed are those of the authors and not necessarily those of the NHS, the NIHR, the Department of Health or other body.

Competing interests All authors have completed the Unified Competing Interests form at http://www.icmje.org/coi_disclosure.pdf (available on request from LH) and declare that LH, AA1, AA2, DKB, AJ, WGJ, SK, GL, CAP, FS, NPW, SF-T, JP, PRH, LS have had no relationships with any companies that might have an interest in the submitted work in the previous 3 years; their spouses, partners and children have no financial relationships that may be relevant to the submitted work; and have no non-financial interests that may be relevant to the submitted work. The exceptions are that.

- The European Hydration Institute (EHI) supplied plane tickets and paid for two nights hotel accommodation for LH to give a talk on dehydration in older people at the EHI's symposium at the International Congress of Nutrition, Granada, Spain 16-18 September 2013;

- The Sjöstrand study was funded by a regional agreement on medical training and clinical research (ALF) between Stockholm County Council and Karolinska Institutet and an unrestricted grant by Masimo Inc, Irvine, California, USA;

- The Fortes study was funded by HydraDx Inc.

Ethics approval The NU-AGE study protocol was approved by the National Research Ethics Committee-East of England (12/EE/0109):
- DRIE was approved by the UK National Research Ethics Service Committee London-East Research Ethics committee (11/L0/1997).

- Fortes study approval was from North West Wales Research Ethics Committee (11/WA/0023).

- Sjöstrand study approval was from Ethical Board of Stockholm County, Sweden (2009/669-31/3).

- Pfortmueller study approval was from the Ethics Committee of the Canton of Bern, Switzerland (14-02-13).

Study procedures for all studies were in accordance with the ethical standards of the Helsinki Declaration. All NU-AGE, Fortes and Sjöstrand participants gave informed consent before participating. DRIE included participants who gave their own informed consent, and also some who were unable to give informed consent (the process of inclusion of these participants was approved by our ethics committee and is explained within this manuscript).

Provenance and peer review Not commissioned; externally peer reviewed.

Data sharing statement No additional data are available.

Open Access This is an Open Access article distributed in accordance with the terms of the Creative Commons Attribution (CC BY 4.0) license, which permits others to distribute, remix, adapt and build upon this work, for commercial use, provided the original work is properly cited. See: http:// creativecommons.org/licenses/by/4.0/

\section{REFERENCES}

1. Siervo M, Bunn D, Prado C, et al. Accuracy of prediction equations for serum osmolarity in frail older people with and without diabetes. Am J Clin Nutr 2014;100:867-76.

2. El-Sharkawy AM, Sahota O, Maughan RJ, et al. Hydration in the older hospital patient-is it a problem? Age Ageing 2014;43:i33-5.

3. Stookey JD, Pieper CF, Cohen HJ. Is the prevalence of dehydration among community-dwelling older adults really low? Informing current debate over the fluid recommendation for adults aged $70+$ years. Public Health Nutr 2005;8:1275-85.

4. Stookey JD. High prevalence of plasma hypertonicity among community-dwelling older adults: results from NHANES III. J Am Diet Assoc 2005;105:1231-9.

5. Bhalla A, Sankaralingam S, Dundas R, et al. Influence of raised plasma osmolality on clinical outcome after acute stroke. Stroke 2000;31:2043-8.

6. Stookey JD, Purser JL, Pieper CF, et al. Plasma hypertonicity: another marker of frailty? J Am Geriatr Soc 2004;52:1313-20.

7. Wachtel TJ, Tetu-Mouradjian LM, Goldman DL, et al. Hyperosmolarity and acidosis in diabetes mellitus: a three-year experience in Rhode Island. J Gen Intern Med 1991;6:495-502.

8. Deardorff DL. Osmotic strength, osmolality, and osmolarity. Am J Hosp Pharm 1980;37:504-9.

9. Thomas DR, Cote TR, Lawhorne L, et al. Understanding clinical dehydration and its treatment. J Am Med Dir Assoc 2008;9:292-301.

10. Naitoh M, Burrell LM. Thirst in elderly subjects. In: Vellas B, Albarede JL, Garry PJ, eds. Hydration and aging. Paris: Serdi, 1998:33-46.

11. Hooper L, Bunn D, Jimoh FO, et al. Water-loss dehydration and aging. Mech Ageing Dev 2014;136-7:50-8.

12. Cheuvront SN, Kenefick RW, Charkoudian N, et al. Physiologic basis for understanding quantitative dehydration assessment. $\mathrm{Am} \mathrm{J}$ Clin Nutr 2013;97:455-62.

13. American Medical Directors Association (AMDA). Dehydration and fluid maintenance in the long-term care setting. Columbia, MD, USA: American Medical Directors Association (AMDA), 2009.

14. Perrier E, Vergne S, Klein A, et al. Hydration biomarkers in free-living adults with different levels of habitual fluid consumption. Br J Nutr 2013;109:1678-87.

15. Johnson EC, Muñoz CX, Le Bellego L, et al. Markers of the hydration process during fluid volume modification in women with habitual high or low daily fluid intakes. Eur J Appl Physiol 2015;115:1067-74.

16. Hooper L, Abdelhamid A, Atreed NJ, et al. Clinical symptoms, signs and tests for identification of impending and current water-loss dehydration in older people. Cochrane Database Syst Rev 2015;4: CD009647.

17. Fortes MB, Raymond-Barker P, Bishop $\mathrm{C}$, et al. Is this elderly patient dehydrated? Diagnostic accuracy of hydration assessment using physical signs, urine, and saliva markers. J Am Med Dir Assoc 2015;16:221-8 
18. Vivanti A, Yu L, Palmer M, et al. Short-term body weight fluctuations in older well-hydrated hospitalised patients. J Hum Nutr Diet 2013;26:429-35.

19. Cheuvront SN, Ely BR, Kenefick RW, et al. Biological variation and diagnostic accuracy of dehydration assessment markers. Am J Clin Nutr 2010;92:565-73

20. Thomas DR, Tariq SH, Makhdomm S, et al. Physician misdiagnosis of dehydration in older adults. J Am Med Dir Assoc 2003:4:251-4.

21. Kyle UG, Bosaeus I, De Lorenzo AD, et al. Bioelectrical impedance analysis-part I: review of principles and methods. Clin Nutr 2004;23:1226-43.

22. Kafri MW, Myint PK, Doherty D, et al. The diagnostic accuracy of multi-frequency bioelectrical impedance analysis in diagnosing dehydration after stroke. Med Sci Monit 2013;19:548-70.

23. Olde Rikkert MGM, van't Hof MA, Baadenhuysen $\mathrm{H}$, et al. Individuality and responsiveness of biochemical indices of dehydration in hospitalized elderly patients. Age Ageing 1998;27:311-19.

24. Institute of Medicine. Panel on dietary reference intakes for electrolytes and water. Dietary reference intakes for water, potassium, sodium, chloride, and sulfate. Washington DC, USA: National Academies Press, 2004.

25. Cheuvront SN, Kenefick RW, Sollanek KJ, et al. Water-deficit equation: systematic analysis and improvement. Am J Clin Nutr 2013;97:79-85.

26. Wikipedia the free encyclopedia. Plasma osmolality. 2014. http:// enwikipediaorg/wiki/Plasma_osmolality [cited 2015 Feb 19].

27. MDcalc. Serum Osmolality/Osmolarity. 2015. http://wwwmdcalccom/ serum-osmolality-osmolarity/\#about-equation [cited 2015 Mar 24]

28. Longmore M, Wilkinson IB, Davidson $\mathrm{EH}$, et al. Clinical Chemistry. In: Oxford handbook of clinical medicine. 8th edn. Oxford: Oxford University Press, 2010:682.

29. Joint British Diabetes Societies. The management of the hyperosmolar hyperglycaemic state (HHS) in adults with diabetes. 2012. http://wwwdiabetesorguk/Documents/Position\%20statements/ JBDS-IP-HHS-Adults.pdf JBDS 06

30. Levey AS, Bosch JP, Lewis JB, et al. A more accurate method to estimate glomerular filtration rate from serum creatinine: a new prediction equation. Modification of Diet in Renal Disease Study Group. Ann Intern Med 1999;130:461-70.

31. Berendson A, Santoro A, Pini A, et al. A parallel randomized trial on the effect of a healthful diet on inflammageing and its consequences in European elderly people: design of the NU-AGE dietary intervention study. Mech Ageing Dev 2013;134:523-30.

32. Santoro A, Pini A, Scurti M, et al. Combating inflammaging through a Mediterranean whole diet approach: the NU-AGE project's conceptual framework and design. Mech Ageing Dev 2014;136-137:3-13.

33. Hooper L, Bunn D. DRIE-Dehydration recognition in our elders. 2014. http://driestudy.appspot.com/

34. Sjöstrand F, Rodhe P, Berglund E, et al. The use of a noninvasive hemoglobin monitor for volume kinetic analysis in an emergency room setting. Anesth Analg 2013;116:337-42.

35. Pfortmueller CA, Wiemann C, Funk G-C, et al. Hypoglycemia is associated with increased mortality in patients with acute decompensated liver cirrhosis. J Crit Care 2014; 29:316.e7-12.

36. Fazekas AS, Funk G-C, Klobassa DS, et al. Evaluation of 36 formulas for calculating plasma osmolality. Intensive Care Med 2013;39:302-8.
37. Zander R. Optimale Berechnung der Osmolalität. Physioklin, Mainz. 2012. http://www.physioklin.de/physiopoc/saeure-basen-sauerstoffelektrolyt-status/optimale-berechnung-der-osmolalitaet.html

38. Kopp JB. Osmolality study.Product literature for Wescor (Logan, UT) vapour pressure ("dew point") osmometer Model 5100. 1973.

39. Gerich JE, Martin MM, Recant L. Clinical and metabolic characteristics of hyperosmolar nonketotic coma. Diabetes 1971;20:228-38.

40. Hoffman RS, Smilkstein MJ, Howland MA, et al. Osmol gaps revisited: normal values and limitations. J Toxicol Clin Toxicol 1993;31:81-93.

41. Koga Y, Purssell RA, Lynd LD. The irrationality of the present use of the osmole gap: applicable physical chemistry principles and recommendations to improve the validity of current practices. Toxicol Rev 2004;23:203-11.

42. Centers for Disease Control and Prevention. National Health and Nutrition Examination Survey. 2015. http://www.cdcgov/nchs/nhanes. htm [cited 2015 Feb 19]

43. Cheuvront SN, Kenefick RW, Heavens KR, et al. A comparison of whole blood and plasma osmolality and osmolarity. J Clin Lab Anal 2014;28:368-73.

44. Zweig MH, Campbell G. Receiver-operating characteristic (ROC) plots: a fundamental evaluation tool in clinical medicine. Clin Chem 1993;39:561-77.

45. Bossuyt PM, Reitsma JB, Bruns DE, et al. Toward complete and accurate reporting of studies of diagnostic accuracy: the STARD initiative. Br Med J 2003;326:41-4.

46. Bland JM, Altman DG. Measuring agreement in method comparison studies. Stat Methods Med Res 1999;8:135-60.

47. Khajuria A, Krahn J. Osmolality revisited-deriving and validating the best formula for calculated osmolality. Clin Biochem 2005;38:514-19.

48. Seifarth CC, Miertschischk J, Hahn EG, et al. Measurement of serum and plasma osmolality in healthy young humans-influence of time and storage conditions. Clin Chem Lab Med 2004:42:927-32.

49. Bohnen N, Terwel D, Markerink M, et al. Pitfalls in the measurement of plasma osmolality pertinent to research in vasopressin and water metabolism. Clin Chem 1992;38:2278-80.

50. Redetzki HM, Hughes JR, Redetzki JE. Differences between serum and plasma osmolalities and their relationship to lactic acid values. Exp Biol Med 1972;139:315-18.

51. Burnett RW, Covington AK, Fogh-Andersen N, et al. Use of ion-selective electrodes for blood-electrolyte analysis. Recommendations for Nomenclature, Definitions and Conventions. International Federation of Clinical Chemistry and Laboratory Medicine (IFCC). Scientific Division Working Group on Selective Electrodes. Clin Chem Lab Med 2000;38:363-70.

52. Dimeski G, Morgan TJ, Presneill JJ, et al. Disagreement between ion selective electrode direct and indirect sodium measurements: estimation of the problem in a tertiary referral hospital. $J$ Crit Care 2012;27:326.e9-16.

53. Edelman IS, Leibman J, O'Meara MP, et al. Interrelations between serum sodium concentration, serum osmolarity, and total exchangeable sodium, total exchangeable potassium, and total body water. J Clin Invest 1958;37:1236-56.

54. Heavens KR, Kenefick RW, Caruso EM, et al. Validation of equations used to predict plasma osmolality in a healthy adult cohort. Am J Clin Nutr 2014;100:1252-6. 